\title{
L'éligibilité des femmes aux droits miniers en République Démocratique du Congo (RDC). Défis et perspectives
}

\author{
Par Genèse Bibi Ekomene
}

\section{Résumé}

Cette étude met l'accent sur la question de l'accès de la femme aux ressources minières en RDC au regard de toutes sortes d'inégalités qu'elle subit, qu'il s'agisse de celle qui œuvre dans les entreprises qui s'en occupent que celles qui sont dans l'exploitation artisanale. Ce constat du traitement fait à la femme, sachant par la nature de chose qu'il ne peut lui être imposé les mêmes conditions que l'homme, a amené l'auteure à s'interroger sur la position du code minier quant à la condition de la femme liée à l'accès aux ressources minières.

Partant de l'analyse des dispositions du code minier et du Règlement minier relatives aux conditions d'éligibilité, l'accès aux ressources minières est reconnu à toute personne tant morale que physique majeure moyennant l'accomplissement des formalités exigées par la loi et les règlements en la matière. Cette reconnaissance ne fait pas une distinction fondée sur le sexe, exception faite de l'hypothèse de l'exclusion de la femme enceinte. Ce qui est compréhensible, puisque considéré comme la protégeant elle et l'enfant des effets toxiques de l'exploitation. Qu'à cela ne tienne, le silence du législateur sur la condition féminine est à la base de beaucoup de discriminations. C'est à titre justement que l'auteure plaide pour la révision du Code minier et Règlement en y insérant des dispositions spécifiquement consacrée à la femme. Ce qui aboutirait à une législation minière séxo-spécifiques et à la promotion du leadership féminin dans ce secteur.

\begin{abstract}
This study focuses on the issue of women's access to mining resources in the DRC in light of all kinds of inequalities that they suffer, whether they work in the companies that deal with them or in artisanal mining. This observation of the treatment of women, knowing by nature that the same conditions cannot be imposed on them as on men, has led the author to question the position of the mining code with respect to the condition of women in relation to access to mining resources.

Based on an analysis of the provisions of the Mining Code and the Mining Regulations relating to eligibility conditions, access to mining resources is recognized for all individuals and legal entities of legal age, provided they comply with the formalities required by the law and the regulations in this area. This recognition does not make a distinction based on gender, with the exception of the exclusion of pregnant women. This is understandable, since it is considered to protect her and her child from the toxic effects of exploitation. Nevertheless, the silence of the legislator on the status of women is the basis of many
\end{abstract}


discriminations. It is precisely for this reason that the author pleads for the revision of the Mining Code and Regulations by inserting provisions specifically devoted to women. This would lead to a specific mining legislation and the promotion of female leadership in this sector.

\section{INTRODUCTION}

Partout ailleurs comme en République Démocratique du Congo (RDC), la jouissance des droits par toutes les personnes et plus particulièrement, par celles dites vulnérables ou vulnérabilisées par nos sociétés, est l'une des manifestations tangibles de l'Etat de droit. Les femmes sont, malheureusement, classées dans ce lot de personnes vulnérables ou vulnérabilisées, notamment par une restriction à l'exercice ou à la jouissance de certains droits, restriction fondée sur le sexe ou à l'absence de la prise en compte des spécificités liées au sexe.

Le secteur minier en RDC paraît un lieu où les droits des femmes ne sont pas respectés tant en ce qui concerne l'exploitation industrielle qu'artisanale. En effet, le secteur minier est régi par la Loi $n^{\circ} 007 / 2002$ du 11 juillet 2002 portant Code minier tel que modifiée et complétée par la Loin ${ }^{\circ} 18 / 001$ du 09 mars $2018^{1}$ et le Décret nº38/2003 du 26 mars 2003 portant Règlement Minier tel que modifié et complété par le Décret $n^{\circ} 18 / 024$ du 08 juin $2018^{2}$ qui fixe les modalités et les conditions d'application de ladite Loi.

Partant du principe de la souveraineté de l'Etat sur les ressources naturelles, l'ensemble de ressources minières telles que définies par les textes en vigueur, se trouvant sur la surface du sol ou renfermés dans le sous-sol ou dans les cours d'eaux du territoire national sont la propriété exclusive, inaliénable et imprescriptible de l'Etat ${ }^{3}$. La propriété étatique sur les ressources naturelles a, entre autres, comme conséquences que les modalités d'accès sont régies par les textes juridiques édictés par les pouvoirs publics.

C'est ainsi que conformément aux dispositions du Code minier, l'accès aux ressources minières (de la recherche à l'exploitation) est reconnu à toutes les personnes tant morales que physiques majeures moyennant l'accomplissement des formalités exigées par la loi minière et les règlements en vigueur. Cette reconnaissance ne fait pas une distinction

1 Loin $^{\circ} 18 / 001$ du 09 mars 2018 modifiant et complétant la loi $\mathrm{N}^{\circ} 007 / 2002$ du 11 juillet 2002 portant code minier, JORDC, $59^{\text {ème }}$ Année, Numéro spécial du 03 mai 2018.

2 Décret $n^{\circ} 038 / 2003$ du 26 mars 2003 portant Règlement Minier tel que modifié et complété par le Décret $n^{\circ} 18 / 024$ du 08 juin 2018, JORDC, $59^{\text {ème }}$ Année, Numéro spécial du 12 juin 2018.

3 Article 9 de la Constitution du 18 février 2006 telle que modifiée par la Loi n $11 / 002$ du 20 janvier 2011, JORDC, $52^{\text {ème }}$ Année, Numéro spécial du 05 février 2011; Joseph CihundaHengelela\& Nicole DjangiEkila, Implication du principe de la souveraineté permanente de l'Etat sur les ressources naturelles sous l'empire de la Constitution du 18 février 2006 en République Démocratique du Congo, disponible sur https://www.nomos-elibrary.de, Consulté le 04 octobre 2020. 
fondée sur le sexe, exception faite de l'hypothèse de l'exclusion de la femme enceinte. Cette exclusion peut se comprendre comme une mesure de protection de la femme ${ }^{4}$.

Le silence du législateur minier sur la situation des femmes contraste avec la situation sur le terrain. Celle-ci indique, en effet, que les femmes, bien minoritaires, sont présentes dans le secteur minier. Elles y sont à la fois comme travailleuses, responsables et membres des Coopératives minières des entreprises minières ainsi que dans les sites miniers artisanaux. En RDC, elles représenteraient près de la moitié des plus de deux millions de

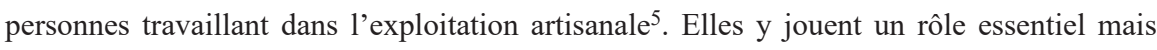
bien différent de celui des hommes.

Cependant, les femmes dans les mines sont victimes de nombreuses discriminations à cause notamment de l'ignorance de leurs droits. Moins de $40 \%$ des femmes sondées savaient que la République démocratique du Congo disposait d'un Code minier, comparativement à $85 \%$ des hommes ${ }^{6}$ et rares sont celles qui disposent d'un titre minier d'exploitation. La situation est plus critique pour les femmes travaillant dans l'artisanat minier ${ }^{7}$. Elles s'adonnent à des travaux comme le concassage, le lavage, le tamisage et le triage ${ }^{8}$ qui les exposent au rayonnement radieux ou à la contraction des maladies du fait de leurs conditions féminines. Plusieurs raisons sont invoquées pour justifier cette répartition genrée du travail: la faiblesse présumée des femmes ou leurs menstruations qui, selon certaines superstitions, les empêcheraient d'être productives. Les tâches de celles qu'on surnomme les " twangaises » sont moins bien rémunérées, même lorsqu'elles exécutent les mêmes fonctions que les hommes ${ }^{9}$.

$\mathrm{Au}$ regard de la situation ainsi décrite des femmes, le Code minier est muet et ne fait aucune distinction entre l'homme et la femme. Bien que les dispositions du code font mention de « toute personne » qui sous-entend l'homme et la femme, il est préférable que des dispositions sexo-spécifiques soient insérées dans le Code minier ou dans le Règlement minier visant à accorder des avantages aux femmes œuvrant dans le secteur minier. Ce qui permettra d'accroître les avantages de leur travail et la réduction des inégalités découlant de l'accès aux ressources minières. L'objectif d'une telle révision législative ou réglementaire

4 Article 5, note 1.

5 Selon les données de la Banque Mondiale. Mais compte tenu de l'informalité du secteur, il est très difficile d'avoir des chiffres précis.

6 Les femmes dans l'exploitation minière artisanale et à petite échelle en RÉPUBLIQUE DÉMOCRATIQUE DU CONGO, disponible sur http://impacttransform.org, Consulté le 20 octobre 2020.

7 Gisèle Eva Côté, Les femmes dans le secteur minier artisanal de l'or en République Démocratique du Congo, rapport du Partenariat Afrique Canada «PAC », publié en novembre 2014, disponible sur https://impacttransform.org, Consulté le 05 octobre 2020.

8 Jeredy Kambasu Malonga, La place de la femme dans l'exploitation minière artisanale dans le territoire de Masisi : Législation et pratique du terrain, travail de recherche publié par Action pour la Liberté et le Développement des Communautés Locales (ADELCO), juin 2017, p.12, disponible sur https://ipisresearch.be, Consulté le 20 octobre 2020.

9 Femme dans les mines : comment extraire l'égalité?, disponible sur https://www.justicepaix.be, Consulté le 15 février 2021. 
est d'assurer l'égalité de sexe et la promotion du genre dans le secteur minier. Ce qui soulève quelques questions : où peut-on situer le silence du législateur par rapport à l'accès de la femme aux ressources minières? Comment interpréter ce silence? Quelles sont les pistes de solutions pour élaguer les écueils au plein accès des femmes dans le secteur minier?

Pour répondre à ces questions, cette étude relève et examine les dispositions du Code minier qui donnent accès aux ressources minières. Il s'agit de celles relatives aux conditions d'éligibilité aux opérations minières (A) et propose de pistes de solution pour y insérer des clauses qui facilitent l'accès et l'épanouissement des femmes dans le secteur minier en RDC (B).

\section{A. LES CONDITIONS D'ELIGIBILITE AUX DROITS MINIERS ET LEUR CARACTERE UNISEXISTE}

Le Code minier a institué un système quasi universel d'accès aux droits miniers en RDC. En effet,

Toute personne morale est autorisée à se livrer à la recherche ou à l'exploitation non artisanale des substances minérales sur toute l'étendue du territoire national, à condition qu'elle soit titulaire d'un droit minier...en cours de validité accordé par l'autorité compétente conformément aux dispositions $d u$....Code. Toute personne physique majeure de nationalité congolaise, excepté la femme enceinte, qui désire se livrer à l'exploitation artisanale des substances minérales sur toute l'étendue du territoire national, ne peut le faire que dans le cadre d'une coopérative minière agréée, conformément aux dispositions du ... Code et dont l'adhésion est subordonnée à la détention d'une carte d'exploitant artisanal ${ }^{10}$.

Il ressort de la lecture des dispositionsqu'il existe des conditions d'éligibilité aux droits miniers qui sont générales (I) et spécifiques à chaque droit minier (II). Il va falloir en saisir la portée.

\section{Conditions générales d'éligibilité aux droits miniers}

Un droit minier est « toute prérogative d'effectuer la recherche et/ou l'exploitation des substances minérales classées en mines » conformément aux dispositions du Code minier. Il peut s'agir du Permis de Recherches (PR), du Permis d'Exploitation (PE), du Permis d'Exploitation des Rejets (PER) et du Permis d'Exploitation de Petite Mine (PEPM). Ces différents permissont qualifiés des droits miniers ${ }^{11}$. Nous avons l'option, dans le cadre de cette réflexion, d'y associer l'exploitation artisanale.

10 Article 5, note 1.

11 Article $1^{\text {er }}$ point 14 , note 1 . 
Suivant les dispositions de l'article 23 du Code minier, les personnes qui en sont éligibles sont de trois ordres. Il s'agit d'abord des personnes morales de droit congolais qui ont leurs sièges sociaux et administratifs sur le territoire national et dont l'objet social porte exclusivement sur les activités minières. Ensuite, toute personne morale de droit étranger dont l'objet social porte exclusivement sur les activités minières et qui se conforme aux lois de la RDC et enfin, tout organisme à vocation scientifique ${ }^{12}$. Ces personnes morales éligibles aux droits miniers sont tenues d'éliredomicile auprès des mandataires en mines établis dans le territoirenational et d'agir par leur intermédiaire.Les personnes morales de droit étranger et les organismes à vocation scientifique ne sont éligibles qu'aux droits miniers de recherches ${ }^{13}$.

Dans la constitution des dossiers de demande des droits miniers, les personnes morales éligibles sont tenues de fournir un certain nombre des documents. Il s'agit d'abord de l'attestation fiscale ou l'équivalent, en cours de validité délivrée par une institution compétente du pays d'origine de la personne morale qui sollicite les droits miniers. Ensuite, de l'attestation de bonne vie et mœurs et l'extrait du casier judiciaire en cours de validité pour les associés de la personne morale, délivrés par les autorités compétentes du pays d'origine et enfin, de l'engagement écrit de déclarer en RDC les profits et revenus réalisés ${ }^{14}$.

D’une manière générale, toute demande de droit minier ne peut être déclarée recevable que si elle remplit les conditions suivantes : l'existence de tous les renseignements requis à l'article 35 du présent Code minier, la production de la preuve du paiement des frais de dépôt ainsi que celle du numéro fiscal, d'identification nationale et du Registre de Commerce et de Crédit Mobilier (RCCM) pour les personnes morales, la conformité de la forme et de la localisation du Périmètre minier, l'existence de l'entièreté du périmètre demandé à l'intérieur du périmètre faisant l'objet du Permis de recherches ${ }^{15}$.

Comme on peut le constater, toutes les conditions générales d'éligibilité aux droits miniers ne contiennent pas de mentions relatives aux femmes. Nulle part, il n'est fait de distinction liée au genre. Toutes les dispositions y relatives sont globales et elles ne font mention que de " toute personne » sans spécifier. Sauf dans l'hypothèse de la femme enceinte qui est exclue de l'exploitation artisanale pendant la période de sa grossesse. Cette exclusion n'est rien d'autres qu'une mesure de protection de la femme et de l'enfant qu'elle porte au regard des risques de toxicité des substances minérales. Qu'en est-il alors des conditions spécifiques?

12 Article 23, note 1.

13 Article 23 alinéa 3, note 1.

14 Article 23 bis, note 1.

15 Article 38 , note 1. 


\section{Conditions spécifiques d'éligibilité à chaque droit minier}

Dans le cadre de cette étude, les droits visés sont les Permis des Recherches (PR), le Permis d'Exploitation (PE), le Permis d'Exploitation des Rejets des Mines (PERM), le droit d'exploitation de la petite mine et le droit d'exploitation artisanale. Ces droits miniers sont constatés par des titres miniers. Il s'agit duCertificat de Recherches, du Certificat d'Exploitation, du Certificat d'Exploitation des Rejets et du Certificat d'Exploitation de Petite Mine.

\section{1) Conditions d'éligibilité au Permis des Recherches (PR)}

La recherche dans le secteur minier est toute activité par laquelle le titulaire d'un droit minier de recherche " se livre, à partir d'indices de l'existence d'un gîte minéral, et au moyen destravaux de surface ou en profondeur, en utilisant notamment des techniques géologiques, géophysiques et géochimiques, y compris diverses méthodes telles que la télédétection, à mettre en évidence l'existence d'un gisement des substances minérales, à le délimiter, et à évaluer la qualité et la quantité des réserves ainsi que les possibilités techniques et commerciales de leur exploitation $»^{16}$.

Pour obtenir le PR, il faut apporter la preuve de la capacité financière et de la compétence technique nécessaires pour mener à bien les recherches afférentes au Permis sollicité et remplir les conditions générales ${ }^{17}$. Aucune exigence n'est requise en rapport avec le genre.

\section{2) Conditions d'éligibilité au Permis d'Exploitation (PE)}

L'exploitation minière est définie comme « toute activité par laquelle une personne morale se livre, à partir d'un gisement identifié, et au moyen des travaux de surface et/ou souterrains, à l'extraction des substances minérales d'un gisement ou d'un gisement artificiel, et éventuellement à leur traitement afin de les utiliser ou de les commercialiser ${ }^{18}$.

Toute personne qui désire obtenir un PE doit adresser une demande au Cadastre Minier (CAMI). Cette demande doit être composée des documents suivants ${ }^{19}$ :

- une copie du Certificat de Recherches en cours de validité;

- le rapport sur le résultat de recherches en ce qui concerne la nature, la qualité, le volume et la situation géographique de la ressource minérale identifiée;

- l'étude de faisabilité de l'exploitation du gisement;

- le plan d'encadrement technique des travaux de développement, de construction et d'exploitation de la mine;

16 Article $1^{\mathrm{er}}$ point 44 , note 1.

17 Article 56, note 1.

18 Article $1^{\mathrm{er}}$ point 20 , note 1 .

19 Article 69, note 1. 
- l'Etude d'Impact Environnemental et Social (EIES) et le Plan de Gestion Environnementale et Sociale (PGES) pour le projet;

- le rapport sur les consultations avec les communautés locales et leurs représentants en application notamment des dispositions de la loi portant principes fondamentaux relatifs à la protection de l'environnement;

- le plan pour la contribution du projet au développement des communautés environnantes;

- le plan de financement avec identification des sources de financement visées, et

- la preuve de paiement des frais de dépôt.

Il importe de relever qu'aucune exigence liée au genre n'est imposée au demandeur dudit PE. Cette même observation est faite pour les conditions qui justifient l'octroi d'un PE à une personne qui l'a demandé. En effet, tout octroi du PE est subordonné aux conditions énumérées à l'article 71 du Code minier. Il s'agit des conditions suivantes :

- démontrer l'existence d'un gisement économiquement exploitable en présentant une étude de faisabilité, accompagnée d'un plan d'encadrement technique des travaux de développement, de construction et d'exploitation de la mine;

- démontrer l'existence des ressources financières nécessaires pour mener à bien son projet selon un plan de financement des travaux de développement, de construction et d'exploitation de la mine ainsi que le plan de réhabilitation du site à sa fermeture.

- obtenir au préalable l'approbation de l'EIES et du PGES du projet;

- céder à l'Etat $10 \%$ des parts ou actions constitutives du capital social de la société requérante;

- créer, à chaque transformation, dans le cadre d'une mine distincte ou d'un projet minier d'exploitation distinct, une société affiliée dans laquelle la société requérante détient au moins $51 \%$ des parts ou actions sociales;

- déposer un acte d'engagement de se conformer au cahier des charges définissant la responsabilité sociétale vis-à-vis des communautés locales affectées par les activités du projet;

- avoir respecté les obligations de maintien de la validité du permis;

- avoirla preuve de la certification de commencement des travaux dûment délivrée par le Cadastre minier;

- avoirla preuve de paiement des droits superficiaires annuels par carré et de l'impôt sur la superficie des concessions minières;

- donner la preuve de la capacité de traiter et de transformer les substances minérales en RDC et déposer un acte d'engagement de traiter et de transformer ces substances sur le territoire congolais, et

- libérer $10 \%$ du capital social en faveur de la participation des personnes physiques de nationalité congolaise dans la société minière ${ }^{20}$.

20 Article 71 bis, note 1. 
Aucune de ces conditions ne fait mention à la femme ou au genre. Qu'en est-il du Permis d'Exploitation des Rejets?

\section{3) Conditions d'éligibilité au Permis d'Exploitation des Rejets des Mines}

L'exploitation des rejets des mines est « toute activité par laquelle un tiers, personne morale, extrait d'un gisement artificiel des substances afin de les traiter éventuellement et de les utiliser ou de les commercialiser $»^{21}$. Outre les conditions générales, le demandeur $\mathrm{du}$ PER doit se soumettre aux exigences de l'instruction à la fois cadastrale, technique et environnementale ${ }^{22}$. Le genre n'apparaît pas non plus ici comme une des conditions d'octroi de ce droit minier.

\section{4) Conditions d'éligibilité au Permis d'exploitation de la petite mine}

L'exploitation minière à petite échelle ou de petite mine est définie comme « toute activité par laquelle une personne morale se livre à une exploitation de petite taille et permanente, exigeant un minimum d'installations fixes, en utilisant des procédés semi-industriels ou industriels, après la mise en évidence d'un gisement $»^{23}$.

L'éligibilité au PEPM est soumise aux mêmes conditions que pour le PE. En outre, personne « ne peut obtenir un Permis d'exploitation de petite mine s'il ne démontre pas l'existence d'un gisement dont les facteurs techniques ne permettent pas une exploitation industrielle rentable en présentant une étude de faisabilité accompagnée d'un plan d'encadrement technique de développement, de construction et d'exploitation de la mine. En plus des conditions énumérées ci-dessus, toute personne de nationalité étrangère doit créer une société de droit congolais en association avec une ou plusieurs personnes de nationalité congolaise dont la participation ne peut être inférieure à $25 \%$ du capital social $»^{24}$.

Ici encore, aucune exigence n'est faite par rapport à la promotion du genre alors qu'il y a une possibilité à travers la participation des personnes de nationalité congolaise.

\section{5) Conditions d'éligibilité à l'exploitation artisanale}

L'exploitation artisanale est définie comme « toute activité par laquelle un exploitant artisanal, se livre, dans une zone d'exploitation artisanale à l'extraction et à la concentration des substances minérales en utilisant des outils, des méthodes et des procédés non industriels... ${ }^{25}$. Seules les personnes physiques majeures de nationalité congolaise détentrices

21 Article $1^{\text {er }}$ point 23, note 1 .

22 Article 91, note 1.

23 Article $1^{\text {er }}$ point 22 , note 1 .

24 Article 104, note 1.

25 Article $1^{\text {er }}$ point 21 , note 1 . 
des cartes d'exploitant artisanal et affiliées aux coopératives minières agréées sont éligibles à l'exploitation artisanale ${ }^{26}$.

La demande de la carte d'Exploitant Artisanal est adressée au Ministre Provincial des Mines et déposée à la Division provinciale des Mines ou au Service des Mines du ressort. Elle est établie sur un formulaire en français ou dans l'une des langues nationales. Le formulaire de demande de carte d'exploitant artisanal comporte les mentions suivantes : l'identité complète, l'adresse et les autres coordonnées du requérant; le niveau d'étude et la profession; l'indication d'un retrait antérieur d'une carte d'exploitant artisanal et, le cas échéant, s'il a suivi un stage de formation en techniques d'exploitation artisanale. A la demande de la carte d'exploitant artisanal doivent être joints les documents suivants : l'engagement de respecter la réglementation en matière de protection de l'environnement, de l'hygiène et de la sécurité dans les zones d'exploitation artisanale, le code de conduite de l'exploitant artisanal comme condition de la validité de sa carte d'exploitant artisanal ${ }^{27}$.

Outre ces conditions d'éligibilité individuelle, il en existe d'autres liées à la coopérative minière. Comme pour les exploitants artisanaux, personnes physiques, les coopératives sont aussi astreintes au respect des normes en matière de sécurité, d'hygiène, d'utilisation de l'eau et de protection de l'environnement ${ }^{28}$. Leur agrément est assujetti au paiement d'un montant fixé par voie règlementaire ${ }^{29}$.

A la demande d'agrément d'une coopérative minière sont annexés les éléments suivants $^{30}$ :

- les statuts dûment notariés de la coopérative d'exploitants artisanaux signés par les fondateurs;

- la liste reprenant les noms et adresses des fondateurs;

- la photocopie certifiée conforme de la carte d'exploitant artisanal de chaque membre;

- le procès-verbal de l'assemblée générale constitutive;

- les noms, adresse et profession des dirigeants;

- la preuve de l'adhésion libre de chaque membre au groupement d'exploitants artisanaux;

- la preuve que les conditions d'adhésion au groupement ne sont pas prohibitives;

- les preuves de versements effectués au titre de souscription au capital social;

- les moyens techniques et financiers ainsi que les ressources humaines que la coopérative entend mettre en œuvre pour la réalisation de ses objectifs.

L'obtention de l'agrément au titre de coopérative minière est subordonnée aux conditions suivantes $^{31}$ :

26 Article 26, note 1.

27 Article 224 du Règlement minier, note 2.

28 Article 112, note 1.

29 Article 114 Bis, note 1.

30 Article 114 Bis, note 1.

31 Article 114 Bis, note 1. 
- être constituée conformément à l'Acte uniforme sur le droit de sociétés coopératives;

- être composée au minimum de vingt personnes physiques majeures de nationalité congolaise détentrices des cartes d'exploitant artisanal des mines valable pour une province donnée, et

- avoir pour objet social, principalement les activités minières.

Pour les autres droits miniers, l'accès à l'exploitation artisanale n'est pas conditionné par un critère de promotion de l'inclusion de la dimension genre dans le secteur minier.

L'analyse des conditions d'octroi des titres miniers laisse entrevoir un silence du législateur minier à l'égard de la question du genre dans le secteur. Comme pour les conditions d'éligibilité, les conditions d'obtention des titres miniers sont générales. Elles ne donnent pas de spécificité sur ce qui concerne l'homme et la femme. Elles n'accordent pas non plus des avantages spécifiques aux femmes, encore moins leur promotion. Nous estimons que le silence du législateur sur la condition de la femme dans l'industrie minière est à la base de différents maux qu'elles subissent. C'est le cas notamment des inégalités dans le traitement, de discrimination et de violence ${ }^{32}$.

Une telle situation est contraire à la Constitution et aux lois de la RDC ainsi qu'aux engagements internationaux. Il importe de signaler, par ailleurs que, c'est depuis 2015 que la RDC s'est assigné les Objectifs du Développement Durable (ODD) qu'il faut atteindre d'ici l'horizon 2030. Parmi ces objectifs, il y a ceux relatifs à l'égalité de sexe, l'autonomisation de la femme et la promotion du leadership féminin. Cette dimension devrait être prise en compte dans tous les domaines notamment dans le secteur minier afin que ces objectifs soient atteints. Le silence sur la promotion de la femme dans le secteur minier dans le chef du législateur minier est un blocage à ce processus de développement durable.

Que faire pour sortir de cette situation? Cette étude propose de lege ferenda quelques pistes de solutions visant à incorporer une dimension sexo-spécifique dans la législation minière congolaise.

\section{B. PERSPECTIVES POUR LA FEMINISATION DES CONDITIONS D'ELIGIBILITE AUX DROITS MINIERS}

A la suite de ce qui précède, il est important de proposer quelques solutions pour un code minier équilibré permettant un accès inclusif de la femme aux opérations minières. Les cibles de ces recommandations sont plus pour les entreprises minières dans l'exploitation industrielle et les exploitants artisanaux étant donné que c'est par ce canal que se fait l'accès aux ressources minières. L'Etat intervient ici en tant que pouvoir régulateur.

32 Rapport alternatif sur l'impact de l'exploitation minière sur les droits des femmes en République démocratique du Congo, publié le 07 juin 2019, p.5, disponible sur https://tbinternet.ohchr.org, Consulté le 05 octobre 2020. 


\section{Pour les entreprises}

Vu que l'Etat congolais n'a pas assez de moyens (matériels et financiers) pour assurer l'exploitation de ces ressources, il l'a confié à des particuliers pour l'exploitation industrielle à travers les entreprises. Le secteur minier Industriel ne s'oppose pas au travail de la femme dans la mine au même titre que dans les zones d'exploitation artisanales. Et pourtant on ne dira pas non plus qu'il offre les mêmes chances d'embauche aux deux sexes. Même à compétence égale, dans le secteur minier, les chances penchent plutôt du côté de l'homme que de la femme ${ }^{33}$. Ces entreprises ne s'intéressent pas très souvent à cette question dans toutes les phases, partant du processus de recrutement des agents jusqu'à leur retraite. La principale raison est que ces entreprises ne sont pas soumises aux mesures contraignantes sur la condition de la femme. Il suffit pour elles de remplir les autres conditions prévues dans le code et les droits de la femme ne constituent pas leur priorité.

Pour ce faire, il revient à l'Etat congolais premièrement d'imposer à ces entreprises d'avoir une politique d'embauche et que cette condition soit insérée dans la Code minier comme l'une des conditions sine qua none d'octroi des titres miniers et/ou des carrières. Deuxièmement, le législateur devrait insérer d'une part, les dispositions protégeant le genre en mettant l'accent sur le critère femme dans le recrutement et la promotion au poste, la formation sur les questions liées au genre, la redistribution des avantages civils, et d'autre part, des dispositions obligeant à ces entreprises de faire un bilan sur le genre à la fin de chaque année.

Avec tous ces détails dans le code, les entreprises seront contraintes de les mettre en pratique de peur de perdre leurs droits miniers. Du point de vue économique, la prise en compte de cet aspect dans le code incite les investisseurs économiques surtout ceux qui s'occupent des droits de la femme.

\section{Pour l'exploitation artisanale}

Les violations des Droits Humains dans le contexte de l'exploitation minière artisanale ne sont pas uniquement des violations des droits substantiels, mais également des violations des droits procéduraux tels que le droit à l'information, à la protection, à la sécurité, à l'éducation etc ${ }^{34}$.Comme il est dit ci-haut, l'exploitation artisanale n'est réservée qu'aux personnes physiques de droit congolais. Dans la pratique, les femmes sont plus visibles sur le terrain mais la plupart sont dans l'informel. L'une des raisons est l'absence de spécification au genre du code. Pour pallier à ce problème, l'Etat congolais doit d'une

33 Micheline KYENGE, Etat des lieux du travail de la femme dans le secteur minier Industriel, disponible sur http://idakdialogue.org, Consulté le 20 octobre 2020.

34 Annie MatunduMbambi, Enquête sur les violations des Droits Humains subies par les femmes congolaises dans l'exploitation des mines artisanales dans la province du Haut Katanga. République Démocratique du Congo, Rapport Women's International League for Peace and Freedom (WILPF/RDC), 2016, disponible sur https://wilpf.org, Consulté le 10 octobre 2020. 
part, favoriser les femmes dans l'exploitation artisanale notamment accorder les zones d'exploitation artisanales aux femmes désirant faire cette opération; d'autre part, il doit appuyer les femmes afin de les aider à se regrouper en coopératives, leur apporter un appui en matériels de protection. Si cet appui est fait au niveau national, cela facilite et encourage l'appui des organisations internationales.

\section{CONCLUSION}

Si de tout temps, la femme a toujours subi toutes sortes d'inégalités et ce, dans tous les secteurs, il faut, malheureusement, constater que la modification du code minier par la Loi de 2018 n'a pas remédié à cette situation. Aucun effet positif n'a été enregistré sur le genre à la suite de cette modification. La question de l'accès de la femme aux ressources minières en RDC ne trouve pas sa place dans la législation y relative. Il y a lieu de constater que les dispositions du code minier précédemment analysées laissent comprendre que les dispositions du code ne sont pasexplicites quant à la condition féminine.

En effet, par la nature, la femme ne peut se voir imposé lesmêmes conditions que l'homme. C'est pour dire qu'aucune proportionnalité ne peut lui être exigée. Actuellement avec la modernité, tous les citoyens doivent être traités sur un même pied d'égalité avec beaucoup plus d'accent sur la promotion du leadership féminin. C'est la prise en compte de tous ces aspects par les textes juridiques nationaux qui renforce un état de droit. La présence de la femme est d'une grande importance dans le secteur minier, surtout qu'elle est déjà dans l'exploitation artisanale bien que la majorité soit dans l'informel. Mais les conditions de son accès ne sont pas favorables. Les sites miniers sont un monde à part, une zone de non-droit et où règne la loi de la jungle ${ }^{35}$.C'est pourquoi nous sommes interrogés sur ce que prévoit le code minier.

Le silence dudit code ne permet pas de protéger la femme. Au contraire, ce silence ouvre beaucoup des portes aux inégalités liées au genre car les dispositions sont globales et ne constituent aucune contrainte. Pour ce faire, l'étude propose de combler le vide constaté par la révision des textes qui régissent la question minière en RDC. Cette proposition de révision est assortie de plusieurs autres recommandations, à savoir :pour les entreprises minières, il revient à l'Etat congolais d'imposer à ces entreprises d'avoir une politique d'embauche et que cette condition soit insérée dans la Code minier comme l'une des conditions sinequanone d'octroi des titres miniers et/ou des carrières. Pour les femmes dans l'artisanat, l'Etat congolais doit d'une part, favoriser les femmes dans l'exploitation artisanale notamment accorder les zones d'exploitation artisanales aux femmes désirant faire cette opération; d'autre part, il doit appuyer les femmes afin de les aider à se regrouper en coopératives, leur apporter un appui en matériels de protection. Celles-cipermettent de contribuer à l'accès inclusif dans le secteur minier et à la promotion du leadership afin de

35 Annie MatunduMbambi et LéonnieKandolo, À l'autre bout de la chaîne : LES FEMMES DANS LES MINES ARTISANALES EN RDC, rapport publié par Women's International League for Peace\&Freedom, août 2016, disponible sur https://wilpf.org, Consulté le 20 octobre 2020. 
produire un code minier qui tienne compte de la condition de la femme et lui accorde des avantages y relatifs.

\section{ANNEXE}

\section{Tableau récapitulatif}

\begin{tabular}{|c|c|}
\hline Types de droitsminiers & Clauses de féminisation \\
\hline Permis des Recherches (PR) & $\begin{array}{l}\text { Obligation à imposer au détenteur du PR à associer les } \\
\text { Congolais, personnes physiques en respectant le genre. } \\
\text { Cette obligation doit se fonder sur la notion de transfert } \\
\text { des compétences }\end{array}$ \\
\hline Permis des Recherches (PR) & $\begin{array}{l}\text { l'Etude d'Impact Environnemental et Social (EIES) et } \\
\text { le Plan de Gestion Environnementale et Sociale (PGES) } \\
\text { pour le projet; } \\
\text { - le rapport sur les consultations avec les communautés } \\
\text { locales et leurs représentants en application notamment } \\
\text { des dispositions de la loi portant principes fondamen- } \\
\text { taux relatifs à la protection de l'environnement; } \\
\text { - le plan pour la contribution du projet au développement } \\
\text { des communautés environnantes; } \\
\text { céder à l'Etat } 10 \% \text { des parts ou actions constitutives du } \\
\text { capital social de la société requérante; } \\
\text { déposer un acte d'engagement de se conformer au ca- } \\
\text { hier des charges définissant la responsabilité sociétale } \\
\text { vis-à-vis des communautés locales affectées par les acti- } \\
\text { vités du projet; } \\
\text { libérer } 10 \% \text { du capital social en faveur de la participa- } \\
\text { tion des personnes physiques de nationalité congolaise } \\
\text { dans la société minière. }\end{array}$ \\
\hline $\begin{array}{l}\text { Permis d'Exploitation des Rejets des } \\
\text { Mines }\end{array}$ & Idem avec le $P E$ \\
\hline $\begin{array}{l}\text { Permis d'exploitation de la petite } \\
\text { mine }\end{array}$ & $\begin{array}{l}\text { En plus des conditions énumérées ci-dessus, toute personne } \\
\text { de nationalité étrangère doit créer une société de droit } \\
\text { congolais en association avec une ou plusieurs personnes } \\
\text { de nationalité congolaise dont la participation ne peut être } \\
\text { inférieure à } 25 \% \text { du capital social }\end{array}$ \\
\hline Exploitation artisanale & \\
\hline
\end{tabular}

\section{BIBLIOGRAPHIE}

\section{TEXTES JURIDIQUES}

- Constitution du 18 février 2006 telle que modifiée par la Loi n¹1/002 du 20 janvier 2011, JORDC, 52 $2^{\text {ème }}$ Année, Numéro spécial du 05 février 2011. 
- Décret n038/2003 du 26 mars 2003 portant Règlement Minier tel que modifié et complété par le Décret $\mathrm{N}^{\circ} 18 / 024$ du 08 juin 2018, JORDC, 59 ème Année, Numéro spécial du 12 juin 2018.

- Loin ${ }^{\circ} 18 / 001$ du 09 mars 2018 modifiant et complétant la loi $\mathrm{N}^{\circ} 007 / 2002$ du 11 juillet 2002 portant code minier, JORDC, $59^{\text {ème }}$ Année, Numéro spécial du 03 mai 2018.

\section{DOCTRINE}

- Joseph CihundaHengelela\& Nicole DjangiEkila, Implication du principe de la souveraineté permanente de l'Etat sur les ressources naturelles sous l'empire de la Constitution du 18 février 2006 en République Démocratique du Congo, disponible sur https://www. nomos-elibrary.de, Consulté le 04 octobre 2020.

\section{AUTRES DOCUMENTS}

- Annie MatunduMbambi, Enquête sur les violations des Droits Humains subies par les femmes congolaises dans l'exploitation des mines artisanales dans la province du Haut Katanga. République Démocratique du Congo, Rapport Women's International League for Peace and Freedom (WILPF/RDC), 2016, disponible sur https://wilpf.org, Consulté le 10 octobre 2020.

- Annie MatunduMbambi et LéonnieKandolo, À l'autre bout de la chaîne : LES FEMMES DANS LES MINES ARTISANALES EN RDC, rapport publié par Women's International League for Peace\&Freedom, août 2016, disponible sur https://wilpf.org, Consulté le 20 octobre 2020.

- Gisèle Eva Côté, Les femmes dans le secteur minier artisanal de l'or en République Démocratique du Congo, rapport du Partenariat Afrique Canada «PAC », publié en novembre 2014, disponible sur https://impacttransform.org, Consulté le 05 octobre 2020.

- Rapport alternatif sur l'impact de l'exploitation minière sur les droits des femmes en République démocratique du Congo, publié le 07 juin 2019, p.5, disponible sur https://tb internet.ohchr.org, Consulté le 05 octobre 2020.

\section{SITES WEBOGRAPHIQUES}

- JeredyKambasuMalonga, La place de la femme dans l'exploitation minière artisanale dans le territoire de Masisi : Législation et pratique du terrain, travail de recherche publié par Action pour la Liberté et le Développement des Communautés Locales (ALDECO), juin 2017, p.12, disponible sur https://ipisresearch.be, Consulté le 20 octobre 2020.

- Micheline KYENGE, Etat des lieux du travail de la femme dans le secteur minier Industriel, disponible sur http://idakdialogue.org, Consulté le 20 octobre 2020. 
- Femme dans les mines : comment extraire l'égalité?, disponible sur https://www.justicep aix.be, Consulté le 15 février 2021.

- Les femmes dans l'exploitation minière artisanale et à petite échelle en RÉPUBLIQUE DÉMOCRATIQUE DU CONGO, disponible sur http://impacttransform.org, Consulté le 20 octobre 2020. 